\title{
Safety and practicability of using mid-upper arm circumference as a discharge criterion in community based management of severe acute malnutrition in children aged 6 to 59 months programmes
}

\author{
Paul J. Binns ${ }^{1 *}$, Nancy M. Dale ${ }^{2}$, Theresa Banda ${ }^{3}$, Chrissy Banda ${ }^{4}$, Bina Shaba ${ }^{5}$ and Mark Myatt ${ }^{6}$
}

\begin{abstract}
Background: The use of proportional weight gain as a discharge criterion for MUAC admissions to programs treating severe acute malnutrition (SAM) is no longer recommended by WHO. The critical limitation with the proportional weight gain criterion was that children who are most severely malnourished tended to receive shorter treatment compared to less severely malnourished children. Studies have shown that using a discharge criterion of MUAC $\geq 125 \mathrm{~mm}$ eliminates this problem but concerns remain over the duration of treatment required to reach this criterion and whether this discharge criterion is safe. This study assessed the safety and practicability of using MUAC $\geq$ $125 \mathrm{~mm}$ as a discharge criterion for community based management of SAM in children aged 6 to 59 months.
\end{abstract}

Methods: A standards-based trial was undertaken in health facilities for the outpatient treatment of SAM in Lilongwe District, Malawi. 258 children aged 6 to 51 months were enrolled with uncomplicated SAM as defined by a MUAC equal or less than $115 \mathrm{~mm}$ without serious medical complications. Children were discharged from treatment as 'cured' when they achieved a MUAC of $125 \mathrm{~mm}$ or greater for two consecutive visits. After discharge, children were followed-up at home every two weeks for three months.

Results: This study confirms that a MUAC discharge criterion of $125 \mathrm{~mm}$ or greater is a safe discharge criterion and is associated with low levels of relapse to SAM (1.9\%) and mortality (1.3\%) with long durations of treatment seen only in the most severe SAM cases. The proportion of children experiencing a negative outcome was $3.2 \%$ and significantly below the $10 \%$ standard ( $p=0.0013$ ) established for the study. All children with negative outcomes had achieved weight-for-height z-score (WHZ) above -1 z-scores at discharge. Children admitted with lower MUAC had higher proportional weight gains $(p<0.001)$ and longer lengths of stay $(p<0.0001)$. MUAC at admission and attendance were both independently associated with cure $(p<0.0001)$. There was no association with negative outcomes at three months post discharge for children with heights at admission below $65 \mathrm{~cm}$ than for taller children ( $p=0.5798)$.

Conclusions: These results are consistent with MUAC $\geq 125 \mathrm{~mm}$ for two consecutive visits being a safe and practicable discharge criterion. Use of a MUAC threshold of $125 \mathrm{~mm}$ for discharge achieves reasonable lengths of stay and was also found to be appropriate for children aged six months or older who are less than $65 \mathrm{~cm}$ in height at admission. Early detection and recruitment of SAM cases using MUAC in the community and compliance with the CMAM treatment protocols should reduce lengths of stay and associated treatment costs.

Keywords: Severe Acute Malnutrition (SAM), Mid Upper Arm Circumference (MUAC), Discharge criteria

* Correspondence: paul@validinternational.org

'Valid International, Oxford, UK

Full list of author information is available at the end of the article 


\section{Background}

The use of mid-upper arm circumference (MUAC) as a general tool (i.e. for case-finding, referral, admission, monitoring response to treatment, and discharge) has the potential to simplify the practical application of community-based management of acute malnutrition (CMAM) services, facilitating their delivery within the framework of primary and community health services.

In 2007 the use of MUAC for case-finding, referral, and admission to CMAM services was accepted by the World Health Organisation (WHO), the World Food Program (WFP), the United Nations Standing Committee on Nutrition (UN/SCN), and the United Nations Children's Fund (UNICEF) [1]. MUAC is now widely used in CMAM programs for these purposes.

The use of proportional weight gain as a discharge criterion for MUAC admissions is no longer recommended by WHO [2], as it had proven to be problematic with the most malnourished cases receiving the least amount of treatment. While the use of MUAC greater than $125 \mathrm{~mm}$ has been used in some settings prior to the WHO updated recommendations and some studies have proposed MUAC as an alternative discharge criterion to percentage weight gain $[3,4]$ for MUAC admissions, the safety of using MUAC greater than $125 \mathrm{~mm}$ as a discharge criterion has not been validated [2].

A consultation between academics and non-governmental organisations (NGOs) in December 2012 [5] concluded that there were concerns over the safety of using MUAC as a discharge criterion due to a lack of evidence with regard to post-discharge relapse and mortality rates. Also, anecdotal evidence suggested that children over six months of age with heights less than $65 \mathrm{~cm}$ admitted with low MUAC would experience very long lengths of stay and may not reach the MUAC discharge threshold within sixteen weeks (112 days) of admission. Published evidence was considered to be inadequate to inform programming.

The aims of this study were to evaluate the safety and practicability of using MUAC as a discharge criterion for children aged 6 to 59 months admitted to outpatient treatment for SAM using MUAC.

\section{Ethics statement}

Written consent was obtained from a parent (or primary caregiver) of the child to be enrolled in the study. Each parent/caregiver signed an individual consent form detailing the proposed treatment, the right to refuse enrolment into the study, the right to confidentiality and anonymity and that no payment would be received for participation.

This study complied with World Medical Association Declaration of Helsinki (Ethical Principles for Medical
Research Involving Human Subjects, 1964) and was approved by the National Health Sciences Research Committee of Malawi (NHSRC, protocol \# 817).

\section{Methods}

The study design was a standards-based trial. There are no internationally agreed standards for post-discharge relapse and mortality in CMAM programs. The standard adopted for the study was informed by a review by Ashworth [6,7], which used a standard of $<10 \%$ relapse as a 'criterion for success' for community-based nutritional rehabilitation programmes prior to the development of the CMAM treatment approach. A standard of less than $10 \%$ for combined post discharge relapse and nonaccidental, non-violent death was adopted for the study through consultation with experts in CMAM programming at FANTA/AED. This standard was more stringent than the standard used by Ashworth. In this article we present estimates for post-discharge relapse and mortality separately and combined so as to allow comparison against other standards.

The study was conducted in five outpatient health facilities run by the Government of Malawi Ministry of Health $(\mathrm{MoH})$ and located in Lilongwe District, Malawi, with care delivered by $\mathrm{MoH}$ staff. A research team from Valid International (comprised of a research coordinator, a registered nurse, and a community mobilisation officer) trained $\mathrm{MoH}$ staff in study protocols and assisted directly in the measurement and treatment of all study subjects. The Malawi National Guidelines for Communitybased Therapeutic Care [8] formed the standard of care. At the start of the study the admission criterion for the treatment of SAM was MUAC $<110 \mathrm{~mm}$ according to the Malawi National guidelines. These guidelines were in the process of being revised to meet the recommendation of the UN Joint Statement of 2009 [2] to raise the admission criteria to MUAC $<115 \mathrm{~mm}$. The clinics in which the study subjects were recruited changed the admission criteria to MUAC $\leq 115 \mathrm{~mm}$ ahead of the publication of the revised guidelines with the consent of the Ministry of Health.

The study enrolled children aged between 6 and 59 months with uncomplicated severe acute malnutrition (SAM) as defined by MUAC $\leq 115 \mathrm{~mm}$ and without medical complications. Children with a MUAC $\leq 115 \mathrm{~mm}$ with concurrent bilateral pitting oedema or medical complications were referred to inpatient care according to Malawi National Guidelines and enrolled into the study on their return from inpatient care.

Subjects were recruited between February 2011 and March 2012. Age was verified by reference to the child's 'health passport'. Children whose caregivers were resident outside of Lilongwe District were excluded from the study but were admitted to the CMAM program and 
provided with standard care according to the Malawi National Guidelines. MUAC, weight and height were measured on admission. An appetite test was conducted to confirm the appropriate consumption of Ready to Use Therapeutic Food (RUTF) which is required in order to receive treatment as an outpatient.

Routine medications given on admission followed the Malawi National Guidelines and included a broad spectrum antibiotic (amoxicillin or co-trimoxazole) and vitamin A. Each child was assessed for malaria using a rapid diagnostic test, $\left(\right.$ Paracheck $\left.\mathrm{Pf}^{\circ}\right)$, and if positive, was treated with antimalarials (lumefantrine and artemether). If the Paracheck $\mathrm{Pf}^{\oplus}$, rapid test was unavailable, the patient was treated empirically for malaria if they presented with a fever of unknown origin. Each caregiver and child was routinely offered serial testing for Human Immunodeficiency Virus (HIV) using the Determine HIV-1/2 Assay ${ }^{\text {Tm }}$ and, if tested positive, Uni-Gold Recombigen HIV assay ${ }^{\text {mix }}$. HIV positive cases were included in the study and referred for appropriate treatment. It was not monitored whether the child went on to receive treatment for HIV. At each visit the child was given a ration of RUTF according to their weight (average 180$200 \mathrm{kcal} / \mathrm{kg} /$ day) and at the second visit anthelminthic treatment (albendazole) was given. The dosage for all medicines was age or weight related and given according to the Malawi National Guidelines.

Study subjects were requested to attend outpatient treatment for SAM on a weekly basis. At each clinic visit the child was measured for weight and MUAC and their appetite for RUTF was assessed. Due to the rotation of $\mathrm{MoH}$ clinical staff in attendance at the health centres, a single observer from the research team conducted each of the measurements of weight, height, and MUAC in order to minimise inter-observer variation. The standardisation of the measurements was assessed using the method of Habicht [9], using the principle investigator as the standard, and was reviewed every 3 months.

If the status of the child had not improved or had deteriorated since the previous visit then the child was referred to the $\mathrm{MoH}$ clinician for further assessment according to Malawi National Guidelines. Any child absent from treatment was followed up at home by a Health Surveillance Assistant (HSA) or communitybased volunteer and encouraged to return for treatment.

Each study subject was discharged 'cured' from outpatient treatment when they achieved a MUAC of $125 \mathrm{~mm}$ or greater on two consecutive clinic visits. On discharge the caregiver was given a fourteen-day supply of RUTF calculated according to the weight of the child. The child was referred to a Supplementary Feeding Program (SFP) if such a program were operating in the health area of residence. A study subject was also discharged from the study if the child died, 'defaulted' (i.e. was absent for three consecutive visits) or was 'noncured' (i.e. had not reached the discharge criteria) after sixteen weeks (112 days). All admissions and discharges from the study were approved and verified by a member of the Valid International research team. Any study subject discharged from the study as 'non-cured' was also assessed according to Malawi National Guidelines and referred back to the standard Ministry of Health programme for further treatment if the child had not reached the discharge criteria as 'cured' according to national guidelines.

Previous studies had indicated that most mortality following treatment for SAM occurs within three months of discharge [10]. Each child that was discharged as 'cured' was followed up every two weeks for a threemonth period by an HSA trained in the study protocols. At each follow-up visit the child was assessed for illness (cough, fever, diarrhoea or vomiting) and their MUAC was measured by the HSA in accordance with their training in the Integrated Management of Childhood Illnesses (IMCI) program. Any child who had relapsed (i.e. met the program admission criteria of MUAC $\leq$ $115 \mathrm{~mm}$ or had bilateral pitting oedema) or had deteriorated clinically was referred back to the health centre for assessment and treatment. Relapsed children were eligible for re-admission to the study if their MUAC was equal to or below $115 \mathrm{~mm}$. Any child absent from their home for two consecutive home visits was verified as being lost to follow-up (LTFU) by consulting with members of the local community regarding the whereabouts of the family. The accuracy of the MUAC measurements and clinical status of the child during follow-up was assessed by a member of the research team. Verification visits were made randomly to children in the follow-up phase of the study and visits were conducted in all cases where relapse was detected or LTFU had occurred.

No preferential treatment was given to study subjects compared with non-study subjects.

\section{Data collection and analysis}

On admission and at each subsequent visit, the child's weight was measured (naked or wearing light undergarments and without shoes) to the nearest $100 \mathrm{~g}$ using Salter $^{\text {rux }}$ scales (Salter-Brecknell 235-6S series), the model used by the health centre for the clinical management of SAM cases. MUAC was measured to the nearest millimetre using a standard non-elastic MUAC tape (UNICEF supply code S0145620 "MUAC, Child 11.5, Red/PAC-50"). On admission and discharge the child's height was measured to the nearest millimetre using a standard paediatric height board (supine length was measured for children less than 24 months of age).

After discharge as 'cured, the child was followed up at home every two weeks for a three-month period by a HSA 
trained in the study protocols. At each follow-up visit the child was assessed for illness (cough, fever, diarrhoea or vomiting) and their MUAC was measured by the HSA.

Data were collected on paper forms designed for the purpose and were entered into an EpiData Version 3.1 (EpiData Association, Odense, Denmark) database using both interactive checking for range and legal values and double-entry validation. Statistical analysis was performed using the $R$ Language for Data Analysis and Graphics [11]. Height-for-age, weight-for-age, and weight-for-height $\mathrm{z}$-scores were calculated according to the WHO Child Growth Standard [12]. The KruskalWallis non-parametric test was used for comparisons of lengths of stay and proportional weight gain by categories of MUAC at admission and categories of height at admission and for comparison of height at admission for 'cured' and 'non-cured' cases.. The null hypothesis for the Kruskal-Wallis test is that the data in each group have the same distribution of the variable of interest. The strength of association between cure and MUAC at admission and attendance rate was investigated using logistic regression.

\section{Results}

Table 1 and 2 describes the study cohort at admission. The study recruited 258 children aged between 6 and 51 months between February 2011 and March 2012 of

Table 1 Main characteristics of study subjects at admission $\left(n=258^{\mathrm{a}}\right)$

\begin{tabular}{lll}
\hline Characteristic & Number & Proportion \\
\hline Males & 115 & $44.6 \%$ \\
Females & 143 & $55.4 \%$ \\
Number with MUAC $\leq 115 \mathrm{~mm}^{\text {b }}$ only & 248 & $96.1 \%$ \\
${\text { Number with MUAC } \leq 115 \mathrm{~mm}^{2} \text { and oedema }}$ & 10 & $3.9 \%$ \\
Requiring inpatient care $^{c}$ & 27 & $10.5 \%$ \\
No concurrent illnesses recorded $^{\text {d }}$ & 125 & $48.4 \%$ \\
Diarrhoea $^{d}$ & 56 & $21.7 \%$ \\
Vomiting $^{d}$ & 23 & $8.9 \%$ \\
Fever $^{d}$ & 65 & $25.2 \%$ \\
Respiratory illness $^{\text {d }}$ & 73 & $28.3 \%$ \\
HIV positive $^{\text {e.f }}$ & 19 & $7.4 \%$ \\
HIV negative $_{\text {HIV status not known (i.e. testing refused) }}$ & 121 & $46.9 \%$ \\
\hline
\end{tabular}

'There were no refusals
${ }^{\mathrm{b}} \mathrm{MUAC}$ tapes were marked in divisions of $1 \mathrm{~mm}$ and color-coded. A'red MUAC',

signifying severe acute malnutrition, was marked red at the $115 \mathrm{~mm}$ cut off point

Inpatient stabilisation care prior to enrolment in the study as per CMAM National Guidelines

${ }^{\mathrm{d}}$ Does not sum to $100 \%$ due to some subjects having multiple illnesses at presentation

${ }^{\mathrm{e}}$ Serial HIV testing according to national protocol using Determine ${ }^{\mathrm{TM}}$ and Uni-Gold Recombigen ${ }^{\text {TM }}$ HIV assays

f Prevalence of HIV in those tested was $13.6 \%$ whom 115 (44.6\%) were male and 143 (55.4\%) were female. The study protocol included children aged up to 59 months but none were older than 51 months. Of the children enrolled in the study, 125 (48.4 \%) did not present with any concurrent illnesses (defined as diarrhoea, vomiting, fever, or acute respiratory symptoms) while $27(10.5 \%)$ presented with complications severe enough to require transfer to inpatient care prior to enrolment in the study. The remaining 106 (41.1\%) of children had concurrent illnesses as outlined in Table 1 and 2. 140 (54.3\%) of children were tested for HIV on admission and of those children, 19 (13.6 \%) of 140 (54.3\%) children were tested for HIV on admission tested positive for HIV.

Figure 1 summarises the anthropometric data of the study subjects at admission in a Venn diagram [13] in terms of MUAC, weight-for-height z-score (WHZ), height-for-age z-score (HAZ) and weight-for-age z-score (WAZ) according to the World Health Organization Growth Standards [12]. Only 4 (1.6\%) of the recruited subjects did not have a WHZ $<-2, \mathrm{HAZ}<-2$, or WAZ $<-2$ on admission. The majority (52.9\%) of the study cohort was undernourished by all three indicators and by MUAC. A large majority (c. $90 \%$ ) of study subjects were stunted.

Table 3 describes the treatment given to the study subjects. A high proportion of subjects $(93.0 \%)$ received antibiotics while $65.0 \%$ received the full national protocol of medicines.

Figure 2 describes the treatment outcomes achieved in the study and the subsequent exclusions from follow-up. $63.2 \%$ of enrolled subjects were cured while $14.0 \%$ defaulted, $3.9 \%$ died during treatment and $19.0 \%$ were discharged as 'non-cured'. The cure rate for the study did not meet the Sphere Minimum Standard of $75 \%$ [14], however, defaulter rates did meet Sphere minimum standards (i.e. less than $15 \%$ ) and the mortality rate was lower than and met the criteria for success set by WHO for community based programmes in non-emergency contexts (i.e. $<5 \%$ ) $[7,15]$. The low cure rate observed was primarily due to children being discharged from the study after 4 months of treatment without attaining the study discharge criteria. Of the 49 cases discharged as 'non-cured' from this study 43 (87.8\%) were discharged as 'cured' according to the existing national guidelines (MUAC > $115 \mathrm{~mm}$ and 2 months minimum stay) while 6 $(12.2 \%)$ required continuing care.

The median attendance rate for 'cured' patients (as a percentage of the total number of weekly visits required of all subjects by the study protocol during treatment) was $87.0 \%$. Figure 3a shows a box plot for the attendance rate for 'cured' and 'non-cured' children and Fig. 3b shows a box plot for the MUAC-at-admission for 'cured' and 'non-cured' children. Attendance and MUAC-at- 
Table 2 Main characteristics of study subjects at admission $\left(n=258^{\mathrm{a}}\right)$

\begin{tabular}{|c|c|c|c|c|c|c|}
\hline Characteristic & Minimum & Lower Quartile & Median & Mean & Upper Quartile & Maximum \\
\hline Age at admission (months) & 6.0 & 10.0 & 14.0 & 16.4 & 21.0 & 51.0 \\
\hline MUAC at admission $(\mathrm{cm})^{c}$ & 8.0 & 10.4 & 11.0 & 10.8 & 11.4 & 11.5 \\
\hline Height at admission (cm) & 53.3 & 62.5 & 67.4 & 67.6 & 72.2 & 92.5 \\
\hline Median duration of inpatient episode (days) & 2.0 & 5.0 & 7.0 & 8.3 & 9.0 & 25.0 \\
\hline
\end{tabular}

aThere were no refusals

${ }^{\mathrm{b}}$ Inpatient stabilisation care prior to enrolment in the study as per CMAM National Guidelines

c101 (39.1\%) of admissions were below $65 \mathrm{~cm}$ in height

admission variables were both independently associated with cure $(p<0.0001)$. Each millimetre increase in admission MUAC resulted in a $2.2 \%(95 \% \mathrm{CI}=1.47 \%$; $2.85 \%)$ increase in the odds of cure. Each percentage point increase in the attendance rate was associated with a $1.13 \%(95 \% \mathrm{CI}=0.89 \% ; 1.38 \%)$ increase in the odds of cure. These increases appear small but they are for small increases in variables with wide ranges. A ten millimetre increase in admission MUAC (e.g. from $102 \mathrm{~mm}$ to $112 \mathrm{~mm}$ ) was associated with a $23.8 \%$ (95\% $\mathrm{CI}=15.7 \%$; $32.4 \%)$ increase in the odds of cure. A twenty-percentage point increase in attendance (e.g. from $60 \%$ to $80 \%$ ) was associated with a $25.2 \%$ (95\% $\mathrm{CI}=19.4 \%$; $31.5 \%)$ increase in the odds of cure.

The 19 children identified as being HIV positive showed similar treatment outcomes to other children: 'Cured' 52.6 \%, 'Died' 5.3 \%, 'Default' $21.1 \%$, and 'Noncured' $15.8 \%$.

Figure 4a shows the distribution of duration of treatment episode while Fig. $4 \mathrm{~b}$ presents box plots describing the length of stay for three classes of MUAC at admission. Figure $4 \mathrm{c}$ shows the distribution of proportional

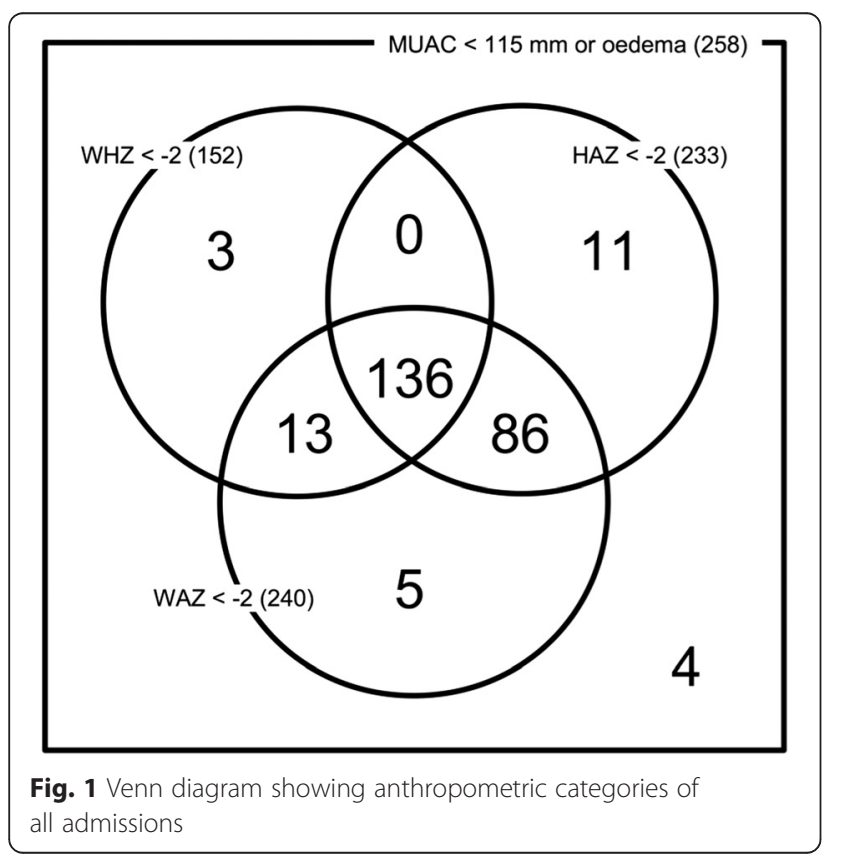

weight gains. Figure $4 d$ presents box plots describing proportional weight gain for three classes of MUAC at admission. The median length of stay of all children in the study was 49 days (IQR $=35$ days -77 days). Children identified as HIV positive and discharged 'cured' had a median duration of treatment of 84 days $(\mathrm{IQR}=$ 63-105 days). Children with lower MUAC at admission required longer durations of treatment (Kruskal-Wallis test, $p<0.0001$ ) with a median duration of treatment in the lowest MUAC group of 98 days (IQR $=60$ days 105 days) and in the highest MUAC group of 42 days (IQR $=35$ days -63 days).

The median proportional weight gain of all children in the study was $21 \%$ (IQR $=16 \%-27 \%)$. Children with lower MUACs experienced higher proportional weight gains (Kruskal-Wallis test, $p<0.0001$ ) with median proportional weight gain of $42 \%$ in the lowest MUAC group (IQR $=40 \%-47 \%)$ and $18 \%$ in the highest MUAC group (IQR $=14 \%-22 \%)$.

Figures $5 \mathrm{a}$ and $\mathrm{b}$ present box plots describing the length of stay and proportional weight gain by height at admission for two height classes at admission respectively. Children recruited to the study with a height at admission below $65 \mathrm{~cm}$ showed a higher proportional weight gain (Kruskal-Wallis test, $p=0.0003$ ) and a longer median length of stay under treatment than taller children (Kruskal-Wallis test, $p=0.0145$ ). These children were no more likely to be discharged as 'non-

Table 3 Treatment given in programme

\begin{tabular}{lll}
\hline Treatment & Number & Percentage \\
\hline RUTF $^{\mathrm{a}}$ & 258 & $100.0 \%$ \\
Vitamin A & 203 & $78.7 \%$ \\
Antimicrobials & 238 & $92.2 \%$ \\
Anthelminthics $^{\mathrm{b}}$ & 108 & $67.1 \%$ \\
Antimalarials & 82 & $31.8 \%$ \\
Received full CMAM protocol $^{\mathrm{c}}$ & 168 & $65.1 \%$ \\
Referred for inpatient care $^{\mathrm{d}}$ & 35 & $13.6 \%$ \\
\hline
\end{tabular}

${ }^{\mathrm{a}}$ Quantity given according to national protocol (average $180-200 \mathrm{kcal} / \mathrm{kg} / \mathrm{day}$ )

${ }^{b}$ Given to eligible children $\geq 12$ months only (according to national protocol).

$79.1 \%$ of all cases received the correct age appropriate intervention

'As per CMAM National Guidelines

${ }^{\mathrm{d} C h i l d r e n}$ referred to inpatient care as per CMAM National Guidelines 


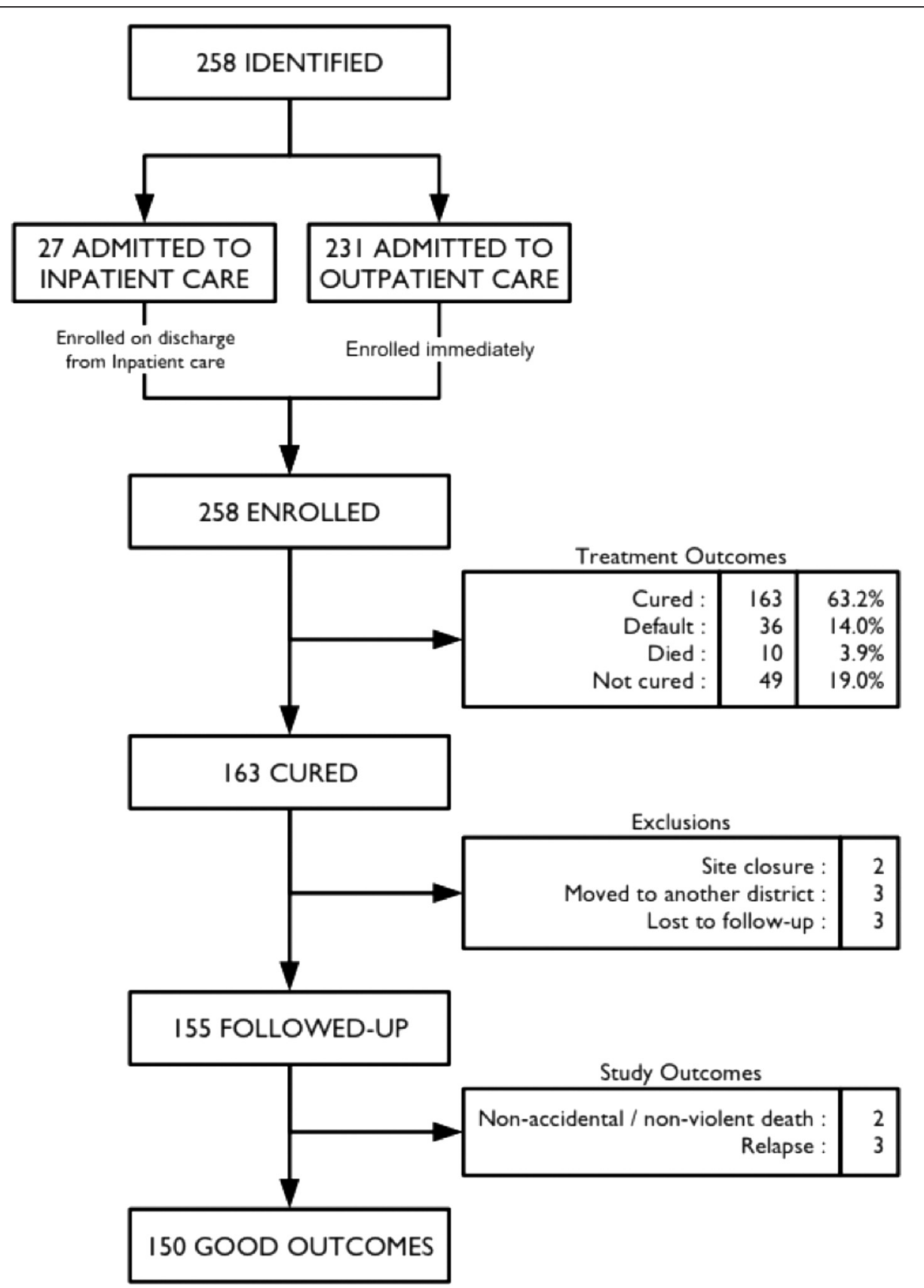

Fig. 2 Flow diagram showing outcomes and exclusions. The study was discontinued at two health centres due to a fall to zero recruitment

cured' than taller children (relative risk $(R R)=0.93$, $95 \% \mathrm{CI}=0.77-1.13$, chi-square $=0.33, \quad p=0.5667)$. There was no association with negative outcomes at three months post discharge for children with heights at admission below $65 \mathrm{~cm}$ than for taller children ( $\mathrm{RR}=1.22,95 \% \mathrm{CI}=0.21-7.09$, Fisher's exact test (single-tailed with $\mathrm{H}_{\mathrm{A}}$ := RR $>1$ ), $p=0.5798$ ).

The median height at admission of 'cured' cases was $67.5 \mathrm{~cm}(\mathrm{IQR}=63.5-72.6)$ and the median height at admission of 'non-cured' cases was $66.5 \mathrm{~cm}$ (IQR =
60.8-70.1). Lower height at admission was not significantly associated with being'non-cured' (Kruskal Wallis test, $p=0.0645)$.

The median height at admission for those known to have died was $62.2 \mathrm{~cm}(\mathrm{IQR}=60.1-65.9)$ and the median height at admission for those not known to have died was $67.5 \mathrm{~cm}(\mathrm{IQR}=63.0-72.3)$. Lower height at admission was significantly associated with mortality (Kruskal Wallis test, $p=0.0369$ ). This analysis should be interpreted with caution as defaulter follow-up was 
a Attendance rate by outcome

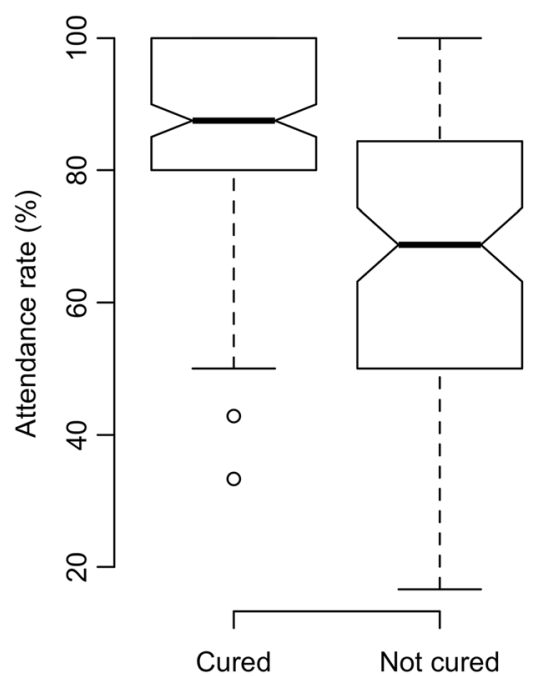

b Admission MUAC by outcome

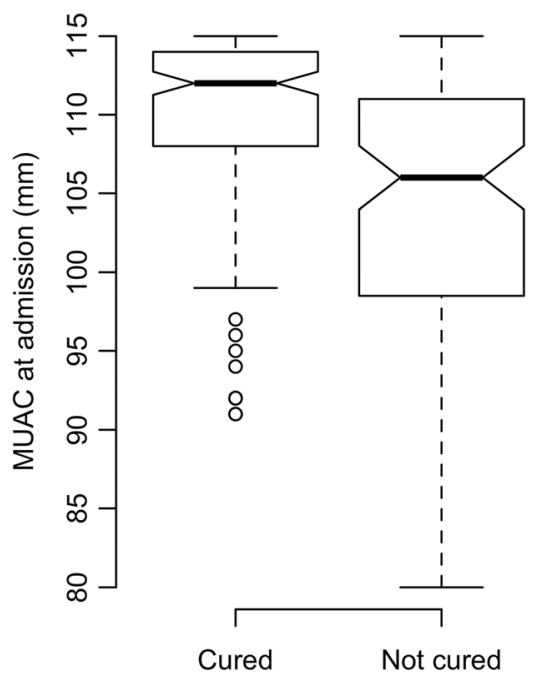

Fig. 3 a Box plot describing Attendance rate for 'cured' and 'non-cured' SAM cases. b: Box plot describing MUAC at admission for 'cured' and 'non-cured' SAM cases

limited and deaths in defaulters may have not been detected and recorded.

Eight children were excluded from the follow-up portion of the analysis. Follow-up was discontinued in these cases due to closure of the study site, relocation of the subject to another district, and inability to be followed because the family were from outside of the health district and had provided an incorrect address upon registration in order to qualify to receive care.

The proportion of patients discharged as 'cured' and experiencing relapse to SAM $(1.9 \%, 95 \% \mathrm{CI}=0.4 \%-$ $5.6 \%)$ or non-violent/non-accidental death (1.3\%, $95 \%$ $\mathrm{CI}=0.2 \%-4.6 \%)$ was $3.2 \%(95 \% \mathrm{CI}=1.1 \%-7.4 \%, p=$ 0.0013 , binomial test with the alternative hypothesis that the proportion of failures is below $10 \%$ ). None of the children identified as HIV positive and discharged 'cured' either 'relapsed' or 'died' within the three-month follow-up period.

Of the 155 children followed up after being discharged as 'cured', $29 \%(n=45)$ receivedSFP rations. There were no negative outcomes amongst these 45 children after three months of follow-up (Risk difference $=4.55 \%$, $95 \% \mathrm{CI}=0.65 \%-8.44 \%$, Fisher one-tailed p-value = $0.1752)$. Of the 45 children receiving SFP, $20 \%(n=9)$ became moderately acutely malnourished (MAM) with a MUAC between 11.5 and $12.4 \mathrm{~cm}$ during the follow-up period. $44 \%(n=4)$ of those who became MAM during follow-up remained MAM at the last follow-up visit.

The study data for the children who 'died' or 'relapsed' are shown in Table 4. A detailed follow-up investigation of the deaths indicated that in both cases the child had accessed medical services at a hospital prior to death.
Regarding relapses, one child was absent for 4 out of the 6 follow-up visits prior to relapse but did not have any illnesses recorded; the second child was recorded to have had diarrhoea, fever and cough in the week prior to relapse and the third child had a cough recorded for weeks 2 and 3 but no other illnesses recorded during the week prior to the relapse being detected in week 5 .

\section{Limitations}

There are several limitations to this study. Occasional interruptions in the $\mathrm{MoH}$ supply of routine medicines lead to shortages of some medicines. When this occurred the $\mathrm{MoH}$ clinician decided on the appropriate use of resources; no priority was given to study subjects. The National Health Sciences Research Committee of Malawi was consulted and ethical approval was given to continue the study despite not all of the study subjects receiving the full CMAM protocol of treatment. The results thus reflect the outcomes achieved in a MoH treatment setting with limited resources.

Following discharge from the study cohort as 'noncured' according to the study criteria, children were assessed for the need for continued treatment or discharged according to the Malawi National guidelines. The compliance and outcome of children who were discharged from the study cohort but who continued treatment or were discharged as 'cured' were not followed up as part of this study.

The limited follow-up of absentees and defaulters during the course of treatment contributed to a reduction in the proportion of the children who were recruited to 
A Length of stay

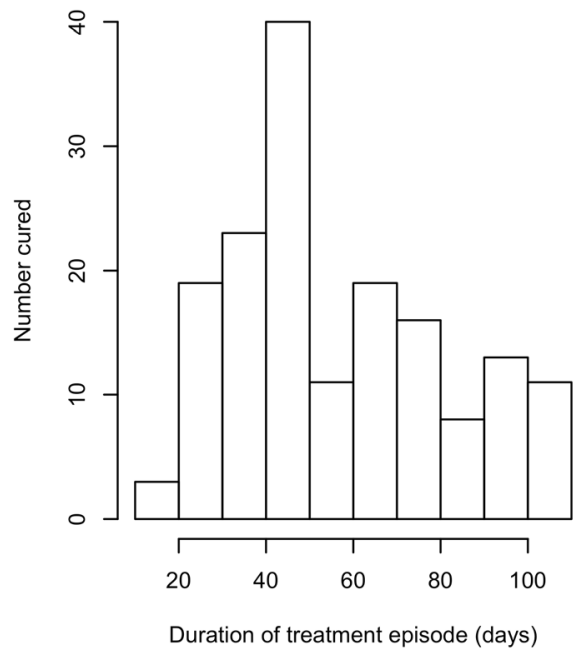

C Proportional weight gain

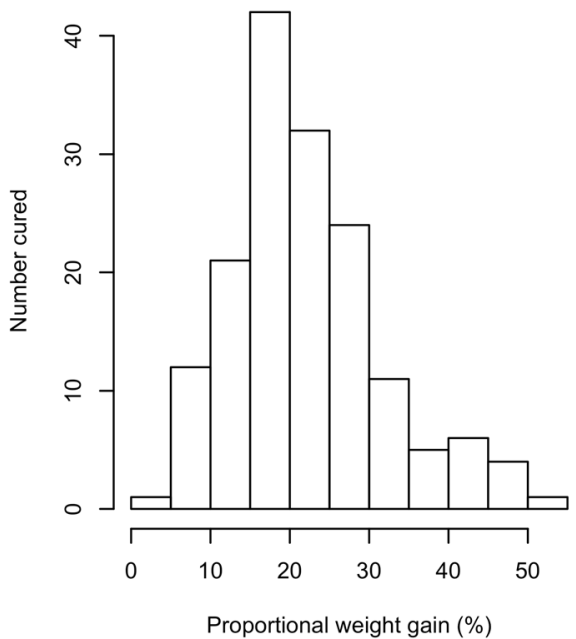

B Length of stay

by MUAC at admission

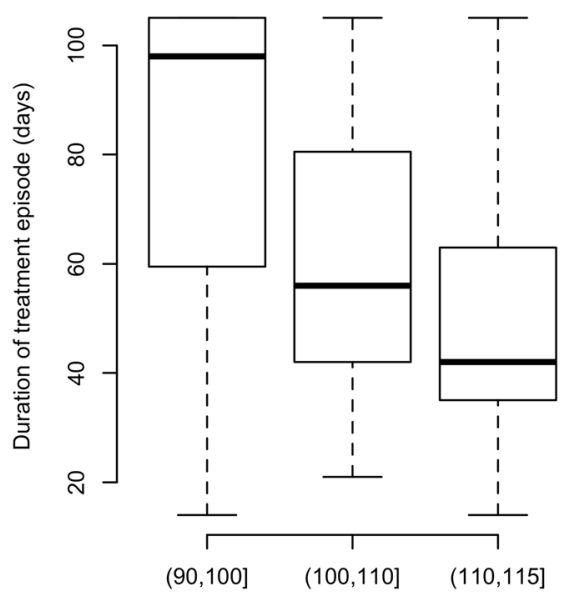

MUAC at admission (mm)

D Proportional weight gain by MUAC at admission

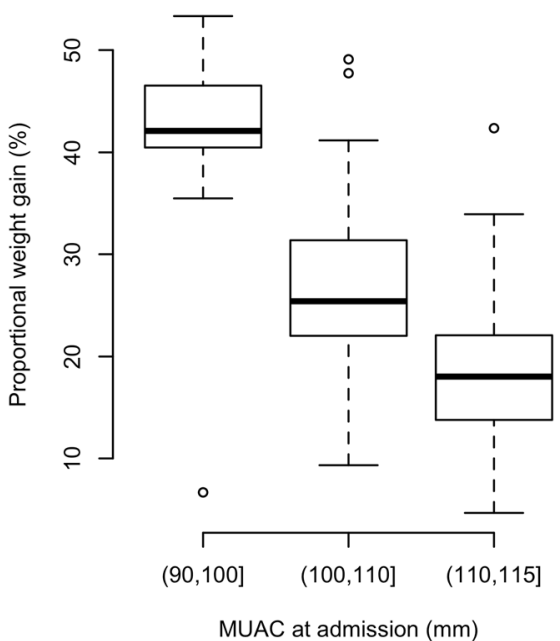

Fig. 4 a Histogram showing distribution of duration of treatment episode. $\mathbf{b}$ : Box plots describing the length of stay for three classes of MUAC at admission $\mathbf{c}$ Histogram showing the distribution of proportional weight gains at discharge. $\mathbf{d}$ Box plots describing proportional weight gain at discharge for three classes of MUAC at admission

the study and later discharged as 'cured', thus limiting the sample size of the study.

The study did not follow compliance with referral for antiretroviral prophylaxis or treatment for HIV for HIV positive cases, however only small differences were observed in programmatic outcomes between HIV positive and HIV negative subjects. The small number of HIV positive subjects did not allow disaggregation of the duration of treatment by MUAC on admission. This issue might be investigated in a larger cohort of HIV positive subjects receiving nutritional rehabilitation prior to starting antiretroviral therapy.
The analyses carried out with this study design were not able to demonstrate that alternative discharge criteria would have resulted in fewer negative outcomes than a discharge criterion of MUAC $\geq 125 \mathrm{~mm}$.

\section{Discussion}

The study confirms that a discharge criterion of MUAC greater than $125 \mathrm{~mm}$ for two consecutive visits represents a practicable and safe discharge criterion according to the standard established for the study and is also an appropriate discharge criterion for children with a height of less than $65 \mathrm{~cm}$ at admission. Relapses formed $1.9 \%$ 
A Length of stay

by height at admission

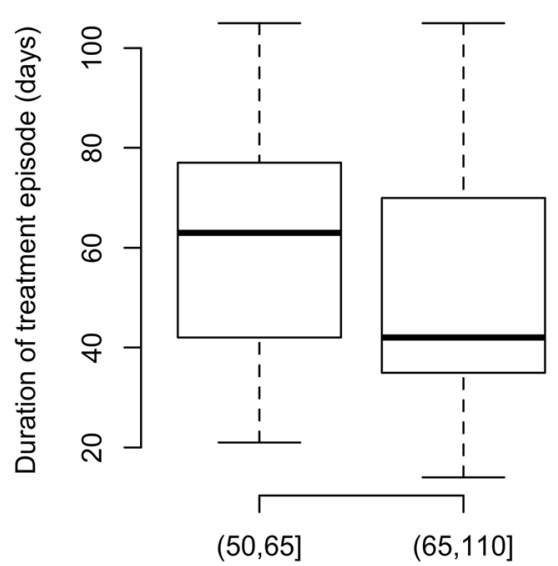

Height at admission (cm)

\section{B Proportional weight gain}

by height at admission

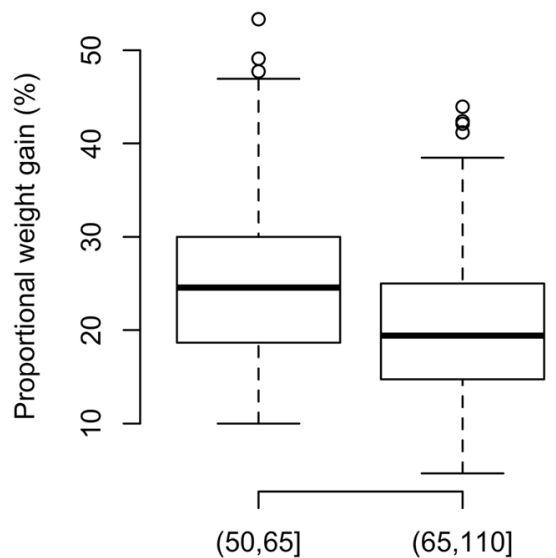

Height at admission (cm)

Fig. 5 a Box plot describing length of stay by height at admission for two height classes. b: Box plot describing proportional weight gain by height at admission for two height classes

(95\% CI $=0.4 \%-5.6 \%$ ) of the cohort of 'cured' patients and non-accidental and non-violent deaths formed $1.3 \%$ (95\% CI $=0.2 \%-4.6 \%$ ) of the cohort of 'cured' patients over the three-month follow-up period. Negative outcomes occurred in $3.2 \%$ (95\% CI $=1.1 \%-7.4 \%)$ of the cohort of 'cured' patients over the three-month followup period. None of the negative outcomes observed during the 3-month follow-up were in children identified as HIV positive at admission. Post-discharge deaths were not associated with relapse to SAM and were not associated with having a discharge WHZ below -2 z-scores. While there are no internationally agreed standards for relapse and mortality following cure from CMAM programmes, all cases with a negative outcome following discharge had a discharge WHZ above -1 z-scores. This suggests that $M U A C \geq 125 \mathrm{~mm}$ for two consecutive visits is as at least safe as discharge using WHZ $>-1 \mathrm{z}$-scores. The rate of relapse and mortality following discharge as 'cured' reported in this study are also similar or lower to those reported by Ashworth in other studies using weight for height (e.g. WHZ > -1 or weight-for-height percentage of median $>85 \%$ ) as the discharge criterion from CMAM programmes [7].

Children with lower MUAC at admission had longer durations of treatment and higher proportional weight gains than the children admitted with a higher MUAC. This shows that the MUAC discharge criterion ensures children who are the most malnourished remain under treatment longer and are discharged with a higher proportional weight gain. This eliminates the problems previously identified with the proportional weight gain criterion [4] and its association with under-treatment for the most

Table 4 Details of study subjects with negative outcomes in the three months following their discharge with a MUAC of $125 \mathrm{~mm}$ or greater $(n=5)$

\begin{tabular}{|c|c|c|c|c|c|c|c|c|c|c|}
\hline \multirow[b]{2}{*}{ Outcome } & \multirow[b]{2}{*}{ Sex } & \multicolumn{2}{|l|}{ Admission } & \multicolumn{4}{|l|}{ Discharge } & \multicolumn{3}{|l|}{ Follow-up } \\
\hline & & Age (months) & $\overline{M U A C}(\mathrm{~mm})^{\mathrm{a}}$ & $\overline{M U A C}(\mathrm{~mm})$ & WHZ & $\begin{array}{l}\text { Length of } \\
\text { stay (days) }\end{array}$ & $\begin{array}{l}\text { Weight } \\
\text { gain (\%) }\end{array}$ & $\begin{array}{l}\text { Duration of } \\
\text { follow-up (weeks) }\end{array}$ & $\begin{array}{l}\text { Last known } \\
\text { MUAC }(\mathrm{mm})^{c}\end{array}$ & $\begin{array}{l}\text { Illness during } \\
\text { follow-up }\end{array}$ \\
\hline Death & $\mathrm{F}$ & 9 & 112 & 125 & +0.30 & 56 & 10.0 & 10 & 134 & diarrhoea cough \\
\hline Death & $\mathrm{F}$ & 14 & 110 & 128 & -0.01 & 105 & 14.7 & 6 & 120 & $\begin{array}{l}\text { diarrhoea vomiting } \\
\text { fever cough }\end{array}$ \\
\hline Relapse & M & 14 & 110 & 126 & -0.08 & 84 & 26.8 & 12 & 110 & none recorded \\
\hline Relapse & M & 12 & 104 & 130 & -0.39 & 35 & 26.8 & 8 & 114 & $\begin{array}{l}\text { diarrhoea vomiting } \\
\text { fever cough }\end{array}$ \\
\hline Relapse & $\mathrm{F}$ & 9 & 110 & 128 & -0.65 & 112 & 30.0 & 6 & 115 & diarrhoea \\
\hline
\end{tabular}

${ }^{a}$ None of the children followed-up had oedema at admission

${ }^{\text {b}}$ The follow-up visit prior to death or the follow-up visit when relapse was detected

'Last known MUAC for deaths is the MUAC recorded in the follow-up visit prior to death. Last known MUAC for relapsed cases is the MUAC at the follow-up visit at which relapse was detected. Relapsed cases were referred back to the therapeutic feeding program 
malnourished individuals. Children identified as HIV positive at admission had a longer median duration of treatment than that observed for all children combined.

Children with height less than $65 \mathrm{~cm}$ at admission also showed greater proportional weight gain and had longer lengths of stay than taller children and showed no association with negative outcomes three months after discharge. These findings may be partly explained due to younger children typically having higher weight gains even when well nourished and that, on average, younger children are shorter. However, these children respond and benefit the most from treatment, and the evidence of this study should allay concerns that using MUAC is not appropriate as an admission or discharge criterion for stunted children aged 6 months or older with a height below $65 \mathrm{~cm}$.

A high proportion of children (92.2\%) received antimicrobials during treatment although the compliance with protocol was lower for Vitamin A and anthelminthics. A low proportion of children (65.1\%) received the full CMAM protocol of medicines. This was due to shortages of supply at the clinics. Whilst this may represent a limitation to this study, the outcomes of the study reflect the context of an $\mathrm{MoH}$ programme with supply shortages.

Reasons for defaulting included waiting times for treatment at the clinic site and prioritisation of household labour (e.g. harvesting). Some defaulters had reportedly migrated away from their temporary home in the treatment catchment area.

It was expected that enrolment into SFP after cure would have a large positive effect on negative outcomes following discharge, however, negative outcomes were rare and any analysis will lack statistical power to detect all but very large effects. The data suggest a small positive effect of SFP enrolment may be present.

Study findings included a high non-cure rate $(18.9 \%)$ for all children combined (15.8 \% for children identified as HIV positive at admission). MUAC at admission and attendance rate were positively associated with cure.

In the absence of the incentivised follow-up of absentees and defaulters; reducing waiting and transit times for clinic visits, frequent counselling to refrain from sharing RUTF, and case review by physicians did little to improve attendance and reduce the non-cure rate over time.

These results provide strong evidence that the proportion 'cured' can be influenced by program design and activities that improve the detection, early recruitment and retention of SAM cases as well as compliance to the SAM treatment protocol.

Care should be taken in extrapolating these results to other populations, however data from CMAM programmes using the same discharge criterion in other contexts report similar findings in terms of median duration of treatment (approximately 8 weeks or 56 days). [3, 4].

Additional studies may be needed to assess whether a discharge criterion of MUAC $\geq 125 \mathrm{~mm}$ is safe in other CMAM programs with low levels of supervision and in other settings. There are currently no internationally agreed standards by which to assess the safety of MUAC or other discharge criteria, however this study indicates that low rates of relapse and mortality following cure are achievable in low resource, non-emergency settings.

\section{Conclusions}

This study confirms that a discharge criterion of MUAC $\geq 125 \mathrm{~mm}$ is safe and practical leading to low mortality and relapse at three months post discharge in the context of Malawian Ministry of Health outpatient care facilities. Negative outcomes were not associated with failing to meet the WHZ $>-2$ z-scores criterion. This MUAC discharge criterion was also found to be appropriate for children aged six months or older who are less than $65 \mathrm{~cm}$ in height at admission. Compliance, as measured by attendance rate, and MUAC at admission were both positively associated with cure.

Children admitted with lower MUAC remain under treatment longer and achieve higher proportional weight gains when MUAC discharge criteria are used. The introduction of MUAC discharge may lead to longer average length of stay initially where there is limited case finding and admission MUAC may be expected to be low. Early detection and recruitment of severe acute malnutrition cases using MUAC in the community and compliance with the CMAM treatment protocols should, however, reduce lengths of stay and associated treatment costs.

\section{Abbreviations \\ AED: academy for educational development; Cl: confidence interval; CMAM: community based management of acute malnutrition; FANTA: food and nutritional technical assistance; FHI: family health international; HIV: human immunodeficiency virus; HSA: health surveillance assistant; LTFU: lost to follow-up; MoH: ministry of health; MUAC: mid upper arm circumference; NGO: non governmental organisation; NHSRC: national health sciences research committee; NRU: nutritional rehabilitation unit; SAM: severe acute malnutrition; UN: United Nations; UNICEF: United Nations children's fund; UNSCN: United Nations standing committee on Nutrition; WFP: world food programme; WHO: world health organisation; WHZ: weight for height z score.}

\section{Competing interests}

Brixton Health is an epidemiological and public health consultancy that works with governments, universities, United Nations organisations, and international NGOs. The company is currently working with MSF, ICRC, ACF, and HelpAge International on developing and testing MUAC straps and bracelets for use with children and adults. This work is being done on a pro-bono-publico basis and the resulting designs will be made available under an open-source public license. Apart from this work there are no competing interests. 


\section{Authors' contributions}

PB contributed to the design, coordination, data collection and statistical data analysis. NMD contributed to the statistical data analysis, TB contributed to the design and coordination. CB contributed to the design, coordination and data collection. BS contributed to the design, coordination and data collection. MM conceived of the study and contributed to the design, coordination and statistical data analysis. All authors helped to draft the manuscript and read and approved the final manuscript.

\section{Acknowledgments}

The authors would like to thank Rebecca Aikman and Kate Sadler of Valid International (UK) and André Briend for proof reading and editing this paper. We would also like to thank Geoffrey Mahone of Valid International (Malawi) for his assistance in data collection. The work described in this report was fully funded by Family Health International (FHI 360) Food and Nutrition Technical Assistance III Project (FANTA-3) as project 4001-08-1-01/4001VALID-00/Task Order 4.

\section{Author details}

'Valid International, Oxford, UK. ²Department for International Health, University of Tampere, Tampere, Finland. ${ }^{3}$ Valid International, Lilongwe, Malawi. ${ }^{4}$ Valid International, Lilongwe, Malawi. ${ }^{5}$ Valid International, Lilongwe, Malawi. ${ }^{6}$ Brixton Health, Llawryglyn, Wales, UK.

Received: 10 June 2015 Accepted: 20 April 2016

Published online: 15 June 2016

\section{References}

1. WHO, WFP, UNSCN, UNICEF. Community-Based Management of Severe Acute Malnutrition: A Joint Statement by the World Health Organization, the World Food Programme, the United Nations System Standing Committee on Nutrition and the United Nations Children's Fund. Geneva: World Health Organization, World Food Programme, United Nations System Standing Committee on Nutrition, United Nations Children's Fund; 2007.

2. WHO. Guideline: Updates on the management of severe acute malnutrition in infants and children. Geneva: World Health Organisation; 2013.

3. Goossens S, Bekele Y, Yun O, Harczi G, Ouannes M, Shepherd S. Mid-upper arm circumference based nutrition programming: evidence for a new approach in regions with high burden of acute malnutrition. PLoS One. 2012;7:e49320.

4. Dale NM, Myatt M, Prudhon C, Briend A. Using mid-upper arm circumference to end treatment of severe acute malnutrition leads to higher weight gains in the most malnourished children. PLoS One. 2013;8:e55404.

5. ENN. Mid-Upper Arm Circumference and Weight-for-Height Z score as indicators of severe acute malnutrition: A consultation of operational agencies and academic specialists to understand the evidence, identify knowledge gaps and to inform operational guidance. 2012.

6. Ashworth A, Community-based rehabilitation of severely malnourished children: a review of successful programmes. London School of Hygiene and Tropical Medicine, 2001: http://imtf.org/_uploads/who-ann-ashworth.pdf

7. Collins S, Sadler K, Dent N, Khara T, Guerrero S, Myatt M, et al. Key issues in the success of community-based management of severe malnutrition. Food Nutrition Bulletin-United Nations University -. 2006;27:S49.

8. Government of Malawi. Ministry of Health. Community based Management of Acute Malnutrition. Malawi National Guidelines; 2007

9. Habicht JP. Standardisation of quantitative epidemiological methods in the field. Bol Officina Sanit Panam May. 1974;76(5):375-84.

10. Roy S K, Chowdury AKMA, Rahaman MM. Excess mortality among children discharged from hospital after treatment for diarrhoea in rural Bangladesh. British Medical Journal, 1983:287;1097-1099

11. Ihaka R, Gentleman R. R: a language for data analysis and graphics. J Computational Graphical Statistics. 1996:5:299-314.

12. De Onis M, WHO. WHO child growth standards: length/height-for-age, weight-for-age, weight-for-length, weight-for-height and body mass index-for-age: methods and development. 2006.
13. Venn J. On the diagrammatic and mechanical representation of propositions and reasonings. London Edinburgh Dublin Philosophical Magazine J Science. 1880;10:1-18.

14. The Sphere Project. humanitarian Charter and Minimum Standards in Humanitarian response, The Sphere Project. UK: Hobbs the printers; 2011.

15. Ashworth A. Efficacy and effectiveness of community-based treatment of severe malnutrition. Food Nutrition Bulletin-United Nations University -. 2006;27:S24.

\section{Submit your next manuscript to BioMed Central and we will help you at every step:}

- We accept pre-submission inquiries

- Our selector tool helps you to find the most relevant journal

- We provide round the clock customer support

- Convenient online submission

- Thorough peer review

- Inclusion in PubMed and all major indexing services

- Maximum visibility for your research

Submit your manuscript at www.biomedcentral.com/submit 